\title{
Numerical Solution of Piecewise Constant Delay Systems Based on a Hybrid Framework
}

\author{
H. R. Marzban and S. Hajiabdolrahmani \\ Department of Mathematical Sciences, Isfahan University of Technology, Isfahan, Iran \\ Correspondence should be addressed to H. R. Marzban; hmarzban@cc.iut.ac.ir
}

Received 5 July 2016; Accepted 22 November 2016

Academic Editor: Jaume Giné

Copyright ( 2016 H. R. Marzban and S. Hajiabdolrahmani. This is an open access article distributed under the Creative Commons Attribution License, which permits unrestricted use, distribution, and reproduction in any medium, provided the original work is properly cited.

\begin{abstract}
An efficient numerical scheme for solving delay differential equations with a piecewise constant delay function is developed in this paper. The proposed approach is based on a hybrid of block-pulse functions and Taylor's polynomials. The operational matrix of delay corresponding to the proposed hybrid functions is introduced. The sparsity of this matrix significantly reduces the computation time and memory requirement. The operational matrices of integration, delay, and product are employed to transform the problem under consideration into a system of algebraic equations. It is shown that the developed approach is also applicable to a special class of nonlinear piecewise constant delay differential equations. Several numerical experiments are examined to verify the validity and applicability of the presented technique.
\end{abstract}

\section{Introduction}

Many problems arising in diverse areas of science and engineering can be described by delay differential equations (DDEs). Typical examples are transmission lines, population dynamics, chemical processes, mathematical biology, physics, electrodynamics, quantum mechanics, robotics, and communication networks [1-3]. Many research works have been devoted to the numerical treatments of DDEs involving constant delay [4-16]. To our knowledge, a few papers have been paid to the numerical investigation of delay differential equations with a piecewise constant delay function [17-21]. It should be mentioned that, except for some special cases, it is either difficult or impossible to obtain a closed-form solution to a constant delay system. Obviously, the situation becomes more complicated when time-delay is a piecewise constant function.

It is generally supposed that the delay function is either constant or continuous. However, in some actual situations, time-delay is a piecewise constant function. Most of the existing computational techniques are capable of providing satisfactory approximation for problems whose solutions are infinitely smooth or well-behaved. To deal with a problem whose solution is a nonsmooth function such as time-delay systems, they encounter some challenges. The computational procedures developed in $[17,18]$ are based on block-pulse functions. Among piecewise constant basis functions, blockpulse functions have a simple structure. Simplicity in operations is a distinctive feature of these functions [22]. As a result, they can be implemented without too much effort. Although these functions possess some nice properties such as disjointedness, orthogonality, and completeness, they do not provide accurate solutions for DDEs. The numerical results obtained by the methods implemented in $[17,18]$ are not in good agreement with the exact solution of the mentioned systems. It should be pointed out that the analytical solution of these systems cannot be produced solely either by continuous basis functions or by piecewise constant basis functions. The main difficulty arises because of the lack of smoothness of the solution associated with DDEs. To overcome these drawbacks, it motivates one to design a flexible framework to accurately model the mixed features of continuity and jumps that occur in the solution of the problem under consideration.

Recently, an effective computational technique for solving DDEs with a piecewise constant delay function has been 
introduced by Marzban and Shahsiah [19]. The developed method is based on a hybrid of block-pulse functions and Chebyshev polynomials. More recently, an efficient numerical scheme has been presented for solving linear piecewise constant delay systems [20]. The proposed approach is based on a hybrid of block-pulse functions and Legendre polynomials. The purpose of this work is to present a simple framework to numerically solve piecewise constant delay systems. The proposed procedure is based on a hybrid of block-pulse functions and Taylor's polynomials. The structure of this kind of hybrid functions is much simpler than that of those available in the literature, particularly, the hybrid of block-pulse functions and Chebyshev polynomials. This type of hybrid functions allows one to significantly reduce the computation time as well as the memory usage. The proposed procedure is also applicable to nonlinear piecewise constant delay systems, but some modifications are required.

The paper is organized as follows. In Section 2, we explain the fundamental properties of the hybrid of blockpulse functions and Taylor's polynomials. In Section 3, the operational matrix of delay associated with the proposed hybrid functions is constructed. Section 4 is dedicated to the problem statement and its description. Finally, in Section 5, various types of piecewise constant delay systems are investigated to evaluate the performance and computational efficiency of the suggested numerical scheme.

\section{Hybrid Functions}

2.1. Hybrid of Block-Pulse Functions and Taylor's Polynomials. Hybrid functions $\phi_{n m}(t), n=1,2, \ldots, N, m=0,1, \ldots, M-1$, are defined on the interval $\left[0, t_{f}\right)$ as $[14]$

$$
\begin{aligned}
& \phi_{n m}(t) \\
& = \begin{cases}T_{m}\left(N t-(n-1) t_{f}\right), & t \in\left[\left(\frac{n-1}{N}\right) t_{f}, \frac{n}{N} t_{f}\right), \\
0, & \text { otherwise, }\end{cases}
\end{aligned}
$$

where $n$ is the order of block-pulse functions. Here $T_{m}(t)=$ $t^{m}, m=0,1, \ldots, M-1$, are the well-known Taylor's polynomials of degree $m$. A detailed explanation of the blockpulse functions can be found in [22]. It is worth noting that the mentioned hybrid functions constitute a semiorthogonal set on the interval $\left[0, t_{f}\right)$.

2.2. Function Approximation. A function $f(t)$ can be represented in terms of the hybrid functions as

$$
f(t)=\sum_{n=1}^{\infty} \sum_{m=0}^{\infty} c_{n m} \phi_{n m}(t)
$$

where the coefficients $c_{n m}, n=1,2, \ldots, N, m=0,1, \ldots, M-$ 1 , are calculated by the following formula:

$$
c_{n m}=\left.\frac{1}{N^{m} m !}\left(\frac{d^{m} f(t)}{d t^{m}}\right)\right|_{t=((n-1) / N) t_{f}} .
$$

If the series given by (2) is truncated, then it can be written in the form

$$
f(t) \simeq \sum_{n=1}^{N} \sum_{m=0}^{M-1} c_{n m} \phi_{n m}(t)=C^{T} \Phi(t)
$$

where

$$
\begin{aligned}
& C=\left[c_{10}, \ldots, c_{1, M-1}, c_{20}, \ldots, c_{2, M-1}, \ldots, c_{N 0}, \ldots, c_{N, M-1}\right]^{T}, \\
& \Phi(t)=\left[\phi_{10}(t), \ldots, \phi_{1, M-1}(t), \phi_{20}(t), \ldots, \phi_{2, M-1}(t), \ldots,\right. \\
& \left.\phi_{N 0}(t), \ldots, \phi_{N, M-1}(t)\right]^{T} .
\end{aligned}
$$

In order to illustrate the usefulness and advantages of the presented framework over the conventional Taylor's expansion, we investigate two cases as follows.

Case 1. Assume that

$$
f(t)= \begin{cases}t, & 0 \leq t<1 \\ 1, & 1 \leq t<2 \\ 3-t, & 2 \leq t \leq 3\end{cases}
$$

Clearly, the function $f(t)$ has two corner points at the points $t=1$ and $t=2$. Accordingly, Taylor's expansion of $f(t)$ about each of the mentioned points does not exist. This function can be exactly approximated by the hybrid functions as follows:

$$
f(t)=\sum_{n=1}^{3} \sum_{m=0}^{1} c_{n m} \phi_{n m}(t)=C^{T} \Phi(t)
$$

in which

$$
\begin{aligned}
C & =\left[0, \frac{1}{3}, 1,0,1,-\frac{1}{3}\right]^{T}, \\
\Phi & (t) \\
& =\left[\phi_{10}(t), \phi_{11}(t), \phi_{20}(t), \phi_{21}(t), \phi_{30}(t), \phi_{31}(t)\right]^{T} .
\end{aligned}
$$

The elements of the vector $\Phi(t)$ are defined by

$$
\begin{aligned}
& \phi_{10}(t)= \begin{cases}1, & t \in[0,1), \\
0, & \text { otherwise }\end{cases} \\
& \phi_{11}(t)= \begin{cases}3 t, & t \in[0,1), \\
0, & \text { otherwise }\end{cases} \\
& \phi_{20}(t)= \begin{cases}1, & t \in[1,2), \\
0, & \text { otherwise }\end{cases}
\end{aligned}
$$




$$
\begin{aligned}
& \phi_{21}(t)= \begin{cases}3 t-3, & t \in[1,2), \\
0, & \text { otherwise }\end{cases} \\
& \phi_{30}(t)= \begin{cases}1, & t \in[2,3), \\
0, & \text { otherwise }\end{cases} \\
& \phi_{31}(t)= \begin{cases}3 t-6, & t \in[2,3), \\
0, & \text { otherwise. }\end{cases}
\end{aligned}
$$

Case 2. Suppose that

$$
g(t)= \begin{cases}\exp (t), & 0 \leq t<1 \\ t^{2}+t, & 1 \leq t<2 \\ t+4, & 2 \leq t \leq 3\end{cases}
$$

Obviously, this function is discontinuous at the points $t=1$ and $t=2$. However, it can be efficiently approximated by the proposed hybrid functions. To express this function in terms of hybrid functions, we choose $N=3$. In addition, Assume that $M$ is an arbitrary positive integer number greater than 2 . With the use of (2), we have

$$
g(t)=\sum_{n=1}^{3} \sum_{m=0}^{M-1} c_{n m} \phi_{n m}(t)
$$

where the hybrid functions $\phi_{n m}(t), n=1,2,3, m=0,1$, $\ldots, M-1$, are defined by

$$
\phi_{n m}(t)=T_{m}(3 t-3 n+3)=3^{m}(t-n+1)^{m} .
$$

Also, the associated coefficients $c_{n m}$ are given by

$$
\begin{aligned}
& c_{1 m}=\frac{1}{3^{m} m !}, \quad m=0,1, \ldots, M-1, \\
& c_{20}=2, \\
& c_{21}=1, \\
& c_{22}=\frac{1}{9}, \\
& c_{2 m}=0, \\
& c_{30}=6, \\
& c_{31}=\frac{1}{3}, \\
& c_{3 m}=0,
\end{aligned}
$$

The hybrid expansion of $g(t)$ over the interval $[1,2]$ is presented by

$$
\begin{aligned}
& c_{20} \phi_{20}(t)+c_{21} \phi_{21}(t)+c_{22} \phi_{22}(t) \\
& =2+(3 t-3)+\frac{1}{9}(3 t-3)^{2}=t^{2}+t .
\end{aligned}
$$

Similarly, the hybrid approximation of $g(t)$ on the interval $[2,3)$ is described by

$$
c_{30} \phi_{30}(t)+c_{31} \phi_{31}(t)=6+\frac{1}{3}(3 t-6)=t+4 .
$$

Moreover, the hybrid expansion of $g(t)$ over the interval $[0,1)$ is expressed by

$$
g(t)=\sum_{m=0}^{M-1} \frac{t^{m}}{m !}
$$

which is precisely Taylor's expansion of the function $\exp (t)$ about $t=0$, up to degree $M-1$. Define

$$
\begin{aligned}
E & =\left\|g(t)-C^{T} \Phi(t)\right\|_{\infty} \\
& =\max \left\{\left|g(t)-C^{T} \Phi(t)\right|: 0 \leq t \leq 1\right\} .
\end{aligned}
$$

It is easily verified that

$$
E=\frac{e}{M !} .
$$

The obtained result indicates that the maximum error converges to zero as the value of $M$ increases. Some important aspects of the proposed hybrid functions are clarified in [23].

2.3. Operational Matrices of Integration and Product. The integration of the vector $\Phi(t)$ presented by (6) can also be expanded in terms of hybrid functions as

$$
\int_{0}^{t} \Phi(\tau) d \tau \simeq P \Phi(t),
$$

in which $P$ is the $M N \times M N$ operational matrix of integration corresponding to the hybrid functions. The structure of this matrix is given by [14]

$$
P=\left(\begin{array}{ccccc}
E^{*} & H & H & \cdots & H \\
0 & E^{*} & H & \cdots & H \\
0 & 0 & E^{*} & \cdots & H \\
\vdots & \vdots & \vdots & \ddots & \vdots \\
0 & 0 & 0 & \cdots & E^{*}
\end{array}\right),
$$

where

$$
H=\frac{1}{N}\left(\begin{array}{cccc}
t_{f} & 0 & \cdots & 0 \\
\frac{t_{f}^{2}}{2} & 0 & \cdots & 0 \\
\vdots & \vdots & \ddots & \vdots \\
\frac{t_{f}^{M}}{M} & 0 & \cdots & 0
\end{array}\right) \text {, }
$$


and $E^{*}$ is defined by

$$
E^{*}=\frac{1}{N}\left(\begin{array}{ccccc}
0 & 1 & 0 & \cdots & 0 \\
0 & 0 & \frac{1}{2} & \cdots & 0 \\
\vdots & \vdots & \vdots & \ddots & \vdots \\
0 & 0 & 0 & \cdots & \frac{1}{M-1} \\
0 & 0 & 0 & \cdots & 0
\end{array}\right) .
$$

It should be noted that $E^{*}$ is the operational matrix of integration associated with the Taylor's polynomials on the interval $\left[((n-1) / N) t_{f},(n / N) t_{f}\right)$. Furthermore, if $C$ is a vector of order $M N \times 1$, then the expression $\Phi(t) \Phi^{T}(t) C$ can be represented in terms of hybrid functions as

$$
\Phi(t) \Phi^{T}(t) C \simeq \widetilde{C} \Phi(t) .
$$

The $M N \times M N$ matrix $\widetilde{C}$ is called the operational matrix of product [14].

\section{Derivation of the Operational Matrix of Delay}

In this section, we shall derive the operational matrix of delay associated with the hybrid of block-pulse functions and Taylor's polynomials. For this purpose, we employ a procedure analogous to the one developed in [19] for the hybrid of block-pulse functions and Chebyshev polynomials. The delayed vector $\Phi(t-a(t))$ can be represented in terms of $\Phi(t)$ as follows:

$$
\Phi(t-a(t))=D \Phi(t),
$$

in which $D$ is called the operational matrix of delay. In addition, $a(t)$ is a piecewise constant function defined by

$$
a(t)= \begin{cases}\tau_{1}, & T_{0} \leq t<T_{1}, \\ \tau_{2}, & T_{1} \leq t<T_{2}, \\ \vdots & \vdots \\ \tau_{r}, & T_{r-1} \leq t<T_{r},\end{cases}
$$

where $0=T_{0}<T_{1}<\cdots<T_{r-1}<T_{r}=t_{f}$. It is further supposed that $\tau_{i}, i=1,2, \ldots, r$, are arbitrary rational numbers in the interval $\left[0, t_{f}\right)$ and $T_{i} \in \mathbb{Q}, i=0,1, \ldots, r$. To construct $D$, we first obtain the value of $N$. Let

$$
A=\left\{i: \tau_{i} \neq 0\right\},
$$

and take $\gamma$ as the smallest positive integer number such that the following conditions are satisfied:

$$
\begin{aligned}
& \gamma \tau_{i} \in \mathbb{Z}, \\
& \gamma T_{j} \in \mathbb{Z}, \\
& \quad i \in A, j=1,2, \ldots, r .
\end{aligned}
$$

Assume that $|A|=k$. Next, we define $\lambda$ as the greatest common divisor of the integers $\gamma \tau_{i}$ and $\gamma T_{j}, i \in A$ and $j=1,2, \ldots, r$; that is,

$$
\lambda=\operatorname{g.c.d}\left(\gamma \tau_{1}, \gamma \tau_{2}, \ldots, \gamma \tau_{k}, \gamma T_{1}, \gamma T_{2}, \ldots, \gamma T_{r}\right) .
$$

Define

$$
N= \begin{cases}\frac{\gamma}{\lambda} t_{f}, & \text { if } \frac{\gamma}{\lambda} t_{f} \in \mathbb{Z}, \\ {\left[\frac{\gamma}{\lambda} t_{f}\right]+1,} & \text { otherwise }\end{cases}
$$

where $\left[(\gamma / \lambda) t_{f}\right]$ denotes the greatest integer value less than or equal to $(\gamma / \lambda) t_{f}$. It is worth noting that $N$ is selected in such a way that the number of subintervals is minimized. As a consequence, we obtain the following subintervals:

$$
\left[0, \frac{\lambda}{\gamma}\right),\left[\frac{\lambda}{\gamma}, 2 \frac{\lambda}{\gamma}\right), \ldots,\left[(N-1) \frac{\lambda}{\gamma}, N \frac{\lambda}{\gamma}\right) .
$$

For convenience, we use the notations defined by

$$
\begin{aligned}
h & =\frac{\lambda}{\gamma}, \\
N_{j} & =\frac{\gamma}{\lambda}\left(T_{j}-T_{j-1}\right), \\
l_{j} & =\frac{\gamma}{\lambda} \tau_{j}, \quad \quad j=1,2, \ldots, r .
\end{aligned}
$$

Clearly $\sum_{j=1}^{r} N_{j}=N$. Consequently, we have

$$
a(t)=k_{i} h, \quad(i-1) h \leq t<i h, i=1,2, \ldots, N,
$$

in which

$$
k_{i}= \begin{cases}l_{1}, & 1 \leq i \leq N_{1}, \\ l_{2}, & N_{1}+1 \leq i \leq N_{1}+N_{2}, \\ \vdots & \vdots \\ l_{j}, & \sum_{k=1}^{j-1} N_{k}+1 \leq i \leq \sum_{k=1}^{j} N_{k}, \\ \vdots & \vdots \\ l_{r}, & \sum_{k=1}^{r-1} N_{k}+1 \leq i \leq \sum_{k=1}^{r} N_{k} .\end{cases}
$$

As a result, the problem is reduced to find the delay operational matrix for the delay function defined by

$$
\Phi(t-a(t))= \begin{cases}\Phi\left(t-k_{1} h\right), & 0 \leq t<t_{1}, \\ \Phi\left(t-k_{2} h\right), & t_{1} \leq t<t_{2}, \\ \vdots & \vdots \\ \Phi\left(t-k_{N} h\right), & t_{n-1} \leq t<t_{N},\end{cases}
$$


where

$$
t_{i}=i h, \quad i=1,2, \ldots, N
$$

To construct the matrix $D$, we first derive the matrix $D_{i}$ for $i=1,2, \ldots, N$, in such a way that the following relation is satisfied:

$$
\Phi\left(t-k_{i} h\right)=D_{i} \Phi(t), \quad t_{i-1} \leq t<t_{i}
$$

By the properties of the hybrid functions, it is easy to verify that, for the case $t_{i-1} \leq t<t_{i}$, the only functions with nonzero values are $\phi_{\left(t-k_{i}\right) m}\left(t-k_{i} h\right)$, for $m=0,1, \ldots, M-1$. Notice that

$$
\phi_{\left(t-k_{i}\right) m}\left(t-k_{i} h\right)=\phi_{i m}(t), \quad m=0,1, \ldots, M-1 .
$$

Accordingly, $\phi_{\left(t-k_{i}\right) m}\left(t-k_{i} h\right)$ can be written in terms of $\phi_{i m}(t)$ as

$$
D_{i}=S_{i} \otimes I_{M}, \quad i=1,2, \ldots, N,
$$

where $I_{M}$ is the $M$-dimensional identity matrix and $\otimes$ denotes the Kronecker product [24]. In addition, $S_{i}$ is an $N \times N$ matrix in which the only nonzero entry is equal to one and located at the $\left(i-k_{i}\right)$ th row and $i$ th column. Finally, if we express $\Phi(t-a(t))$ in terms of $\Phi(t)$, we find

$$
D=D_{1}+D_{2}+\cdots+D_{N}
$$

Remark 1. If $i-k_{i} \leq 0$, then $S_{i}$ is a zero matrix of order $N \times N$. It is worth noting that the structure of the operational matrix of delay associated with the proposed hybrid functions is exactly the same as the structure of the hybrid of blockpulse functions and Chebyshev polynomials.

\section{Problem Statement and Its Approximation}

Consider the time-varying piecewise constant delay system described by

$$
\begin{aligned}
& \dot{X}(t)=E(t) X(t)+F(t) X(t-a(t))+G(t) U(t), \\
& X(0)=X_{0}, \\
& X(t)=0, \quad t<0, \\
& a(t)= \begin{cases}\tau_{1}, & T_{0} \leq t<t_{1}, \\
\tau_{2}, & T_{1} \leq t<T_{2}, \\
\vdots & \vdots \\
\tau_{r}, & T_{r-1} \leq t<T_{r},\end{cases}
\end{aligned}
$$

where $X(t) \in \mathbb{R}^{l}, U(t) \in \mathbb{R}^{q}, E(t), F(t)$, and $G(t)$ are matrices of appropriate dimensions, $X_{0}$ is a constant specified vector, and $\tau_{i}, i=1,2, \ldots, r$, are arbitrary rational numbers in $\left[0, t_{f}\right)$. The objective is to find $X(t), 0 \leq t \leq t_{f}$, satisfying (41)-(43).
4.1. Description of the Method. Let

$$
\begin{aligned}
& X(t)=\left[x_{1}(t), x_{2}(t), \ldots, x_{l}(t)\right]^{T}, \\
& U(t)=\left[u_{1}(t), u_{2}(t), \ldots, u_{q}(t)\right]^{T} .
\end{aligned}
$$

Assume that each $x_{i}(t)$ and each of $u_{j}(t), i=1,2, \ldots, q$, can be written in terms of hybrid functions as

$$
\begin{aligned}
& x_{i}(t)=\Phi^{T}(t) X_{i}, \\
& u_{j}(t)=\Phi^{T}(t) U_{j} .
\end{aligned}
$$

Consequently

$$
\begin{aligned}
& X(t)=\left(I_{l} \otimes \Phi^{T}(t)\right) X, \\
& U(t)=\left(I_{q} \otimes \Phi^{T}(t)\right) U,
\end{aligned}
$$

where $I_{l}$ and $I_{q}$ are identity matrices of order $l \times l$ and $q \times$ $q$, respectively. Furthermore, $X$ and $U$ are vectors of order $l N M \times 1$ and $q N M \times 1$, respectively, given by

$$
\begin{aligned}
& X=\left[X_{1}^{T}, X_{2}^{T}, \ldots, X_{l}^{T}\right]^{T}, \\
& U=\left[U_{1}^{T}, U_{2}^{T}, \ldots, U_{q}^{T}\right]^{T} .
\end{aligned}
$$

Also

$$
X(0)=\left(I_{l} \otimes \Phi^{T}(t)\right) d,
$$

where $d$ is a vector of order $l N M \times 1$, defined by

$$
d=\left[d_{1}^{T}, d_{2}^{T}, \ldots, d_{l}^{T}\right]^{T} .
$$

Similarly, $E(t), F(t)$, and $G(t)$ can be represented in terms of hybrid functions as

$$
\begin{aligned}
& E(t)=E^{T}\left(I_{l} \otimes \Phi(t)\right), \\
& F(t)=F^{T}\left(I_{l} \otimes \Phi(t)\right), \\
& G(t)=G^{T}\left(I_{q} \otimes \Phi(t)\right),
\end{aligned}
$$

where $E^{T}, F^{T}$, and $G^{T}$ are of dimensions $l \times l N M, l \times l N M$, and $l \times q N M$, respectively. The delayed vector $X(t-a(t))$ can also be expressed in terms of hybrid functions as

$$
X(t-a(t))=\left(I_{l} \otimes \Phi^{T}(t)\right)\left(I_{l} \otimes D^{T}\right) X,
$$

in which $D$ is the delay operational matrix presented by (25). As a result

$$
\begin{aligned}
& E(t) X(t)=E^{T}\left(I_{l} \otimes \Phi(t)\right)\left(I_{l} \otimes \Phi^{T}(t)\right) X \\
& \quad=\left(I_{l} \otimes \Phi^{T}(t)\right) \widetilde{E}^{T} X, \\
& F(t) X(t-a(t)) \\
& \quad=F^{T}\left(I_{l} \otimes \Phi(t)\right)\left(I_{l} \otimes \Phi^{T}(t)\right)\left(I_{l} \otimes D^{T}\right) X \\
& \quad=\left(I_{l} \otimes \Phi^{T}(t)\right) \widetilde{F}^{T}\left(I_{l} \otimes D^{T}\right) X,
\end{aligned}
$$




$$
\begin{aligned}
& G(t) U(t)=G^{T}\left(I_{q} \otimes \Phi(t)\right)\left(I_{q} \otimes \Phi^{T}(t)\right) U \\
& =\left(I_{l} \otimes \Phi^{T}(t)\right) \widetilde{G}^{T} U,
\end{aligned}
$$

where $\widetilde{E}, \widetilde{F}$, and $\widetilde{G}$ can be determined in a manner analogous to the derivation process of the matrix $\widetilde{C}$ described by (24). Furthermore

$$
\begin{aligned}
& \int_{0}^{t}\left(I_{l} \otimes \Phi^{T}(s)\right) d s=\left(I_{l} \otimes \Phi^{T}(t)\right)\left(I_{l} \otimes P^{T}\right), \\
& \int_{0}^{t} F(s) X(s-a(s)) d s \\
& \quad=\left(I_{l} \otimes \Phi^{T}(t)\right)\left(I_{l} \otimes P^{T}\right) \widetilde{F}^{T}\left(I_{l} \otimes D^{T}\right) X,
\end{aligned}
$$

in which $P$ is the operational matrix of integration described by (20). By integrating both sides of (41) with respect to $t$ and using (47), (49), (51), (52), and (54), we obtain

$$
\begin{aligned}
\left(I_{l} \otimes\right. & \left.\Phi^{T}(t)\right) X-\left(I_{l} \otimes \Phi^{T}(t)\right) d \\
= & \left(I_{l} \otimes \Phi^{T}(t)\right)\left(I_{l} \otimes P^{T}\right) \widetilde{E}^{T} X \\
& +\left(I_{l} \otimes \Phi^{T}(t)\right)\left(I_{l} \otimes P^{T}\right) \widetilde{F}^{T}\left(I_{l} \otimes D^{T}\right) X \\
& +\left(I_{l} \otimes \Phi^{T}(t)\right)\left(I_{l} \otimes P^{T}\right) \widetilde{G}^{T} U .
\end{aligned}
$$

From the preceding equation, we deduce that

$$
\begin{aligned}
X & =\left[I_{M N}-\left(I_{l} \otimes P^{T}\right) \widetilde{E}^{T}\right. \\
& \left.-\left(I_{l} \otimes P^{T}\right) \widetilde{F}^{T}\left(I_{l} \otimes D^{T}\right)\right]^{-1}\left[d+\left(I_{l} \otimes P^{T}\right) \widetilde{G}^{T} U\right] .
\end{aligned}
$$

\section{Simulation Results}

In this section, five examples of varying complexity are investigated to evaluate the usefulness and effectiveness of the proposed computational technique. The correct choice of $N$ would greatly improve the efficiency and accuracy of the presented procedure.

5.1. Example 1. Consider the following piecewise constant delay system [17]:

$$
\begin{aligned}
& \dot{x}(t)=x(t-a(t))+u(t), \quad 0 \leq t \leq 1, \\
& x(t)=0, \quad t \leq 0, \\
& u(t)=1, \quad t \geq 0, \\
& a(t)= \begin{cases}0.1, & 0 \leq t<0.35, \\
0.3, & 0.35 \leq t<0.7, \\
0.5, & 0.7 \leq t \leq 1 .\end{cases}
\end{aligned}
$$

The exact solution to this problem is given by

$$
\begin{aligned}
& 0 \leq t<0.1, \\
& 0.1 \leq t<0.2, \\
& 0.2 \leq t<0.3, \\
& 0.3 \leq t<0.35, \\
& 0.35 \leq t<0.4, \\
& 0.4 \leq t<0.5, \\
& 0.5 \leq t<0.6, \\
& 0.6 \leq t<0.65, \\
& 0.65 \leq t<0.7, \\
& 0.7 \leq t<0.8, \\
& 0.8 \leq t<0.85, \\
& 0.85 \leq t<0.9, \\
& 0.9 \leq t \leq 1 .
\end{aligned}
$$


To apply the method developed in the current paper, we first choose the value of $N$, with the use of (30). Because $\gamma=$ 20 and $\lambda=1$, hence, we select $N=20$. We also take $M=6$. Let

$$
x(t)=C^{T} \Phi(t)
$$

where

$$
\begin{aligned}
& C=\left[c_{10}, \ldots, c_{1, M-1}, \ldots, c_{N 0}, \ldots, c_{N, M-1}\right]^{T}, \\
& \Phi(t)=\left[\phi_{10}(t), \ldots, \phi_{1, M-1}(t), \ldots, \phi_{N 0}(t), \ldots,\right. \\
& \left.\quad \phi_{N, M-1}(t)\right]^{T} .
\end{aligned}
$$

By expanding $u(t)$ in terms of hybrid functions, we get

$$
u(t)=d_{1}^{T} \Phi(t)
$$

We also have

$$
x(t-a(t))=C^{T} D \Phi(t),
$$

where $D$ is the delay operational matrix given by (25). Integrating both sides of (57) with respect to $t$ and using (62)(65) yield

$$
C^{T}=d_{1}^{T} P\left(I_{M N}-D P\right)^{-1},
$$

in which $I_{M N}$ is the $M N$-dimensional identity matrix and $P$ is the operational matrix of integration given by (20). With the use of (66) and (62), we would obtain the same value as the exact value of $x(t)$.

5.2. Example 2. Consider the following two-dimensional time-varying piecewise constant delay system:

$$
\begin{aligned}
\left(\begin{array}{l}
\dot{x}_{1}(t) \\
\dot{x}_{2}(t)
\end{array}\right)= & \left(\begin{array}{cc}
1 & 1+t \\
2 t & t^{2}
\end{array}\right)\left(\begin{array}{l}
x_{1}(t-a(t)) \\
x_{2}(t-a(t))
\end{array}\right) \\
& +\left(\begin{array}{l}
1 \\
2
\end{array}\right) u(t), \quad 0 \leq t \leq 1, \\
\left(\begin{array}{l}
x_{1}(t) \\
x_{2}(t)
\end{array}\right)= & \left(\begin{array}{l}
0 \\
0
\end{array}\right), \quad t<0, \\
\left(\begin{array}{l}
x_{1}(0) \\
x_{2}(0)
\end{array}\right)= & \left(\begin{array}{l}
1 \\
1
\end{array}\right), \\
u(t)= & \begin{cases}t, & 0 \leq t<0.5, \\
1-t, & 0.5 \leq t<1,\end{cases} \\
a(t)= & \begin{cases}0.2, & 0 \leq t<0.3, \\
0.7, & 0.3 \leq t \leq 1 .\end{cases}
\end{aligned}
$$

The exact solutions to this problem are given as follows:

$$
x_{1}(t)=\left\{\begin{array}{lr}
1+\frac{1}{2} t^{2}, & 0 \leq t<0.2, \\
\frac{4319}{7500}+\frac{103}{50} t+\frac{18}{25} t^{2}+\frac{11}{30} t^{3}+\frac{1}{4} t^{4}, & 0.2 \leq t<0.3, \\
\frac{147071}{120000}+\frac{1}{2} t^{2}, & 0.3 \leq t<0.5, \\
\frac{117071}{120000}+t-\frac{1}{2} t^{2}, & 0.5 \leq t<0.7, \\
-\frac{10331}{12000}+\frac{747}{200} t-\frac{161}{200} t^{2}+\frac{1}{30} t^{3}+\frac{1}{4} t^{4}, & 0.7 \leq t<0.9, \\
-\frac{424558459}{4200000000}+\frac{1148571}{500000} t-\frac{355883}{2000000} t^{2}-\frac{4681}{30000} t^{3}+\frac{1767}{4000} t^{4}+\frac{71}{750} t^{5}-\frac{7}{120} t^{6}+\frac{1}{35} t^{7} & 0.9 \leq t \leq 1, \\
x_{2}(t)=\left\{\begin{array}{lr}
1+t^{2}, & 0 \leq t<0.2, \\
\frac{179473}{187500}+\frac{101}{50} t^{2}+\frac{16}{75} t^{3}+\frac{3}{20} t^{4}+\frac{1}{5} t^{5}, & 0.2 \leq t<0.3, \\
\frac{3169351}{3000000}+t^{2}, & 0.3 \leq t<0.5, \\
\frac{1669351}{3000000}+2 t-t^{2}, & 0.5 \leq t<0.7, \\
-\frac{220481}{3000000}+2 t+\frac{49}{200} t^{2}+\frac{3}{100} t^{3}-\frac{1}{10} t^{4}+\frac{1}{5} t^{5}, & 0.7 \leq t<0.9, \\
\frac{36460339381}{84000000000}+2 t-\frac{63163}{40000} t^{2}+\frac{4372217}{3000000} t^{3}-\frac{11101}{40000} t^{4}+\frac{1981}{15000} t^{5}+\frac{191}{900} t^{6}-\frac{11}{140} t^{7}+\frac{1}{40} t^{8}, & 0.9 \leq t \leq 1 .
\end{array}\right.
\end{array}\right.
$$


To solve this problem by the proposed approach, we first determine the value of $N$ with the use of (30). Because $\gamma=10$ and $\lambda=1$, consequently, we select $N=10$. Also, we choose $M=9$. Let

$$
\begin{aligned}
& x_{1}(t)=C_{1}^{T} \Phi(t), \\
& x_{2}(t)=C_{2}^{T} \Phi(t) .
\end{aligned}
$$

Expanding 1, $1+t, 2 t$, and $t^{2}$ in terms of hybrid functions implies

$$
\begin{array}{r}
1=f_{1}^{T} \Phi(t), \\
1+t=f_{2}^{T} \Phi(t), \\
2 t=f_{3}^{T} \Phi(t), \\
t^{2}=f_{4}^{T} \Phi(t) .
\end{array}
$$

Also, using (70), we obtain

$$
\begin{aligned}
& \int_{0}^{t} u(\tau) d \tau \\
& \quad= \begin{cases}\frac{1}{2} t^{2}=f_{5}^{T} \Phi(t), & 0 \leq t<0.5, \\
-\frac{1}{4}+t-\frac{1}{2} t^{2}=f_{6}^{T} \Phi(t), & 0.5 \leq t<1 .\end{cases}
\end{aligned}
$$

Moreover

$$
\begin{aligned}
& x_{1}(t-a(t))=C_{1}^{T} D \Phi(t), \\
& x_{2}(t-a(t))=C_{2}^{T} D \Phi(t) .
\end{aligned}
$$

Therefore

$$
\begin{aligned}
(1+t) x_{2}(t-a(t)) & =C_{2}^{T} D \Phi(t) \Phi^{T}(t) f_{2} \\
& =C_{2}^{T} D \tilde{f}_{2} \Phi(t) \\
2 t x_{1}(t-a(t)) & =C_{1}^{T} D \Phi(t) \Phi^{T}(t) f_{3} \\
& =C_{1}^{T} D \tilde{f}_{3} \Phi(t) \\
t^{2} x_{2}(t-a(t)) & =C_{2}^{T} D \Phi(t) \Phi^{T}(t) f_{4} \\
& =C_{2}^{T} D \tilde{f}_{4} \Phi(t)
\end{aligned}
$$

in which $\widetilde{f}_{2}, \widetilde{f}_{3}$, and $\widetilde{f}_{4}$ can be obtained in a way similar to the construction method of the matrix $\widetilde{C}$ defined by (24). By integrating both sides of (67) with respect to $t$ and using (68), (70), (71), (73), (74), (75), (76), and (77), we get

$$
\begin{gathered}
C_{1}^{T}\left(I_{M N}-D P\right)-C_{2}^{T} D \widetilde{f}_{2} P=f_{1}^{T}+f_{5}^{T}+f_{6}^{T}, \\
C_{2}^{T}\left(I_{M N}-D \widetilde{f}_{4} P\right)-C_{1}^{T} D \widetilde{f}_{3} P=f_{1}^{T}+2 f_{5}^{T}+2 f_{6}^{T} .
\end{gathered}
$$

By solving the resulting system described by (78) and then using (73), we would obtain the same values as the exact values of $x_{1}(t)$ and $x_{2}(t)$.

5.3. Example 3. Consider the following time-delay system [3]:

$$
\begin{aligned}
& \dot{x}(t)=-5 x(t)-5 x(t-a(t))+2 u(t), \quad 0 \leq t \leq 2, \\
& x(0)=1, \\
& u(t)=1, \quad t \geq 0,
\end{aligned}
$$

$$
a(t)= \begin{cases}0, & 0 \leq t<0.8 \\ 0.3, & 0.8 \leq t<1.4 \\ 0.6, & 1.4 \leq t \leq 1.7 \\ 0.9, & 1.7 \leq t \leq 2\end{cases}
$$

The exact solution to this problem is given by [3]

$$
x(t)= \begin{cases}0.2+0.8 e^{-10 t}, & 0 \leq t<0.8 \\ 0.2+0.8 e^{3-10 t}+0.8 e^{-4}\left(1-e^{3}\right) e^{-5 t} & 0.8 \leq t<1.1 \\ 0.2+0.8 e^{5-10 t}-4 e^{-2.5}\left(1-e^{3}\right) t e^{-5 t}+0.8\left(1-e^{3}\right)\left(6.5 e^{-2.5}+e^{-4}\right) e^{-5 t}, & 1.1 \leq t<1.4 \\ 0.2+0.8 e^{9-10 t}-4 e^{-1}\left(1-e^{3}\right) t e^{-5 t}+0.8\left(1-e^{3}\right)\left(8 e^{-1}-0.5 e^{-2.5}+e^{-4}\right) e^{-5 t}, & 1.4 \leq t<1.7 \\ 0.2+0.8 e^{12-10 t}-4 e^{0.5}\left(1-e^{3}\right) t e^{-5 t}+0.8\left(1-e^{3}\right)\left(9.5 e^{0.5}-0.5 e^{-1}-0.5 e^{-2.5}+e^{-4}\right) e^{-5 t}, & 1.7 \leq t \leq 2 .\end{cases}
$$

To employ the proposed method, we first determine the suitable value of $N$. Using (30), it follows that

$$
N=\frac{\gamma}{\lambda} t_{f}=20
$$

Assume that $M$ is an arbitrary positive integer number. Let

$$
x(t)=C^{T} \Phi(t)
$$


Similarly

$$
\begin{aligned}
x(0) & =u(t)=d^{T} \Phi(t), \\
x(t-a(t)) & =C^{T} D \Phi(t) .
\end{aligned}
$$

By integrating both sides of (79) with respect to $t$ and using (85)-(86), we conclude that

$$
C^{T}=\left(d^{T}+2 d^{T} P\right)\left(I_{M N}+5 P+5 D P\right)^{-1},
$$

where $I_{M N}$ is the $M N$-dimensional identity matrix. Define

$$
E=\max \left\{\left|x_{e}(t)-x(t)\right|: 0 \leq t \leq 1\right\},
$$

in which $x(t)$ and $x_{e}(t)$ denote the approximate solution determined by the present approach and the exact solution, respectively. In Table 1 , the numerical results of the maximum error denoted by $E$, for $N=20$, and various values of $M$ are reported. The simulation results obtained by the current approach show a significant improvement in the accuracy of the solution compared to the method developed in [19].
5.4. Example 4. As a more complicated problem, consider the following problem:

$$
\dot{x}(t)=t x^{2}(t-a(t))+x^{3}(t-a(t))+u(t),
$$

$$
0 \leq t \leq 1 \text {, }
$$

$$
\begin{aligned}
& x(t)=0, \quad t<0, \\
& x(0)=1,
\end{aligned}
$$

$$
\begin{aligned}
& u(t)= \begin{cases}t, & 0 \leq t<0.4, \\
0.4, & 0.4 \leq t \leq 1,\end{cases} \\
& a(t)= \begin{cases}0.4, & 0 \leq t<0.8, \\
0.8, & 0.8 \leq t \leq 1 .\end{cases}
\end{aligned}
$$

The analytical solution to this problem is described by

$$
x(t)= \begin{cases}1+\frac{1}{2} t^{2}, & 0 \leq t<0.4 \\ \frac{663251}{1640625}+\frac{25933}{15625} t-\frac{729}{6250} t^{2}+\frac{117}{250} t^{3}-\frac{3}{100} t^{4}+\frac{13}{100} t^{5}-\frac{1}{120} t^{6}+\frac{1}{56} t^{7}, & 0.4 \leq t<0.8 \\ \frac{235691}{1640625}+\frac{42187}{15625} t-\frac{7623}{6250} t^{2}+\frac{253}{250} t^{3}-\frac{43}{100} t^{4}+\frac{23}{100} t^{5}-\frac{7}{120} t^{6}+\frac{1}{56} t^{7}, & 0.8 \leq t \leq 1 .\end{cases}
$$

Although the above-mentioned problem is a nonlinear delay differential equation, the method developed in the present paper can easily be implemented. Because $\gamma=5$ and $\lambda=2$, we take $N=3$. Furthermore, we take $M=8$. Let

$$
x(t)=C^{T} \Phi(t)
$$

Similarly

$$
\begin{aligned}
x(0) & =v_{1}^{T} \Phi(t), \\
t & =v_{2}^{T} \Phi(t), \\
\int_{0}^{t} u(\tau) d \tau & = \begin{cases}\frac{1}{2} t^{2}=v_{3}^{T} \Phi(t), & 0 \leq t<0.4, \\
-\frac{2}{25}+\frac{2}{5} t=v_{4}^{T} \Phi(t), & 0.4 \leq t<1,\end{cases}
\end{aligned}
$$

where $v_{1}, v_{2}, v_{3}$, and $v_{4}$ are vectors of order $M N \times 1$. Also, we have

$$
\begin{aligned}
x(t-a(t)) & =C^{T} D \Phi(t), \\
x^{2}(t-a(t)) & =C^{T} D \Phi(t) \Phi^{T}(t) D^{T} C \\
& =C^{T} D \widetilde{W} \Phi(t),
\end{aligned}
$$

$$
\begin{aligned}
x^{3}(t-a(t)) & =C^{T} D \widetilde{W} \Phi(t) \Phi^{T}(t) W \\
& =C^{T} D \widetilde{W}^{2} \Phi(t),
\end{aligned}
$$

in which

$$
W=D^{T} C \text {. }
$$

Using (99) gives

$$
\begin{aligned}
t x^{2}(t-a(t)) & =C^{T} D \widetilde{W} \Phi(t) \Phi^{T}(t) v_{2} \\
& =C^{T} D \widetilde{W} \widetilde{v}_{2} \Phi(t)
\end{aligned}
$$

Integrating both sides of (89) with respect to $t$ and using (95), (97), (98), (99), (100), and (102) imply that

$$
\begin{aligned}
& C^{T}\left(I_{M N}-D \widetilde{W} \widetilde{v}_{2} P-D \widetilde{W}^{2} P\right)-\left(v_{1}^{T}+v_{3}^{T}+v_{4}^{T}\right) \\
& \quad=0 .
\end{aligned}
$$

By solving (103) and using (95), the exact value of $x(t)$ will be obtained. 
TABLE 1: Computational results of maximum error for Example 3.

\begin{tabular}{lcc}
\hline Present method & CPU time $(\mathrm{s})$ & $E$ \\
\hline$N=20, M=1$ & 0.112 & $1.165 e-02$ \\
$N=20, M=2$ & 0.202 & $3.231 e-03$ \\
$N=20, M=4$ & 0.398 & $2.573 e-06$ \\
$N=20, M=6$ & 0.769 & $4.836 e-08$ \\
$N=20, M=8$ & 0.853 & $3.942 e-11$ \\
$N=20, M=10$ & 1.282 & $2.376 e-13$ \\
$N=20, M=12$ & 1.841 & $5.875 e-16$ \\
$N=20, M=14$ & 2.167 & $3.624 e-17$ \\
$N=20, M=16$ & 2.536 & $5.117 e-20$ \\
\hline
\end{tabular}

5.5. Example 5. As the final example, we consider the timevarying multidelay system defined by

$$
\begin{aligned}
\dot{x}(t)=t x\left(t-a_{1}(t)\right)+t^{2} x^{2}\left(t-a_{2}(t)\right)+u(t), & \\
& 0 \leq t \leq 1,
\end{aligned}
$$

$$
x(0)=1,
$$$$
u(t)= \begin{cases}t, & 0 \leq t<\frac{1}{2} \\ \frac{1}{2}, & \frac{1}{2} \leq t \leq 1\end{cases}
$$

where

$$
\begin{aligned}
& a_{1}(t)= \begin{cases}\frac{1}{2}, & 0 \leq t<\frac{3}{4}, \\
\frac{1}{4}, & \frac{3}{4} \leq t \leq 1,\end{cases} \\
& a_{2}(t)= \begin{cases}\frac{1}{4}, & 0 \leq t<\frac{1}{2}, \\
\frac{3}{4}, & \frac{1}{2} \leq t \leq 1\end{cases}
\end{aligned}
$$

The analytical solution to this problem is described by

$$
x(t)= \begin{cases}1+\frac{1}{2} t^{2}, & 0 \leq t<\frac{1}{4}, \\ \frac{20373}{20480}+\frac{1}{2} t^{2}+\frac{11}{32} t^{3}-\frac{1}{16} t^{4}+\frac{1}{10} t^{5}, & \frac{1}{4} \leq t<\frac{1}{2}, \\ \frac{48191}{61440}+\frac{1}{2} t+\frac{9}{16} t^{2}-\frac{1}{6} t^{3}+\frac{1}{8} t^{4}, & \frac{1}{2} \leq t<\frac{3}{4}, \\ \frac{1289743}{1966080}+\frac{1}{2} t+\frac{14287}{40960} t^{2}+\frac{187}{384} t^{3}-\frac{1}{256} t^{4}+\frac{1}{24} t^{5}+\frac{1}{48} t^{6}, & \frac{3}{4} \leq t \leq 1 .\end{cases}
$$

Although the above-mentioned system involves multiple piecewise constant delays, the proposed procedure can be applied. To employ our approach, we select $N=4$. By choosing $M=7$, the exact solution of $x(t)$ will be obtained.

\section{Conclusion}

An efficient and flexible framework has been successfully developed for solving piecewise constant delay systems. The foundation of the proposed method is based on a hybrid of block-pulse functions and Taylor's polynomials. The operational matrix of delay has been constructed. The operational matrices of integration, delay, and product associated with the hybrid functions were utilized to transform the problem under consideration into a system of algebraic equations. It is worth emphasizing that the exact solutions of Examples $1,2,4$, and 5 cannot be produced solely either by blockpulse functions or by Taylor's polynomials. After determining the suitable value of $N$, the number of subintervals, small values for $M$, the degree of the Taylor's polynomials, are needed to achieve a specific accuracy. The simulation results demonstrate the effectiveness of the suggested procedure. In addition, the proposed numerical scheme can be extended to a class of nonlinear piecewise constant delay systems, but some modifications are required.

\section{Competing Interests}

The authors declare that there is no conflict of interests regarding the publication of this manuscript.

\section{References}

[1] R. D. Driver, Ordinary and delay differential equations, SpringerVerlag, New York, NY, USA, 1977.

[2] M. Malek-Zavarei and M. Jamshidi, Time-delay systems, vol. 9 of North-Holland Systems and Control Series, North-Holland Publishing Co., Amsterdam, Netherlands, 1987.

[3] K. B. Datta and B. M. Mohan, Orthogonal functions in systems and control, vol. 9 of Advanced Series in Electrical and Computer Engineering, World Scientific Publishing Co., 1995.

[4] A. Bellen, "One-step collocation for delay differential equations," Journal of Computational and Applied Mathematics, vol. 10, no. 3, pp. 275-283, 1984.

[5] C. Hwang and M. Y. Chen, "Analysis and parameter identification of time-delay systems via shifted Legendre polynomials," International Journal of Control, vol. 41, no. 2, pp. 403-415, 1985. 
[6] I. R. Horng and J. H. Chou, "Analysis, parameter estimation and optimal control of time-delay systems via Chebyshev series," International Journal of Control, vol. 41, no. 5, pp. 1221-1234, 1985.

[7] A. Bellen and M. Zennaro, "Numerical solution of delay differential equations by uniform corrections to an implicit Runge-Kutta method," Numerische Mathematik, vol. 47, no. 2, pp. 301-316, 1985.

[8] I.-R. Horng and J.-H. Chou, "Analysis and parameter identification of time-delay systems via shifted Jacobi polynomials," International Journal of Control, vol. 44, no. 4, pp. 935-942, 1986.

[9] M. Razzaghi and M. Razzaghi, "Taylor series analysis of timevarying multi-delay systems," International Journal of Control, vol. 50, no. 1, pp. 183-192, 1989.

[10] B. M. Mohan and K. B. Datta, "Analysis of linear time-invariant time-delay systems via orthogonal functions," International Journal of Systems Science, vol. 26, no. 1, pp. 91-111, 1995.

[11] A. Bellen and S. Maset, "Numerical solution of constant coefficient linear delay differential equations as abstract Cauchy problems," Numerische Mathematik, vol. 84, no. 3, pp. 351-374, 2000.

[12] M. Razzaghi and H. R. Marzban, "A hybrid domain analysis for systems with delays in state and control," Mathematical Problems in Engineering, vol. 7, no. 4, pp. 337-353, 2001.

[13] H. R. Marzban and M. Razzaghi, "Solution of time-varying delay systems by hybrid functions," Mathematics and Computers in Simulation, vol. 64, no. 6, pp. 597-607, 2004.

[14] H. R. Marzban and M. Razzaghi, "Analysis of time-delay systems via hybrid of block-pulse functions and Taylor series," Journal of Vibration and Control, vol. 11, no. 12, pp. 1455-1468, 2005.

[15] H. R. Marzban and M. Razzaghi, "Solution of multi-delay systems using hybrid of block-pulse functions and Taylor series," Journal of Sound and Vibration, vol. 292, no. 3-5, pp. 954-963, 2006.

[16] H. R. Marzban, "Optimal control of linear multi-delay systems based on a multi-interval decomposition scheme," Optimal Control Applications and Methods, vol. 37, no. 1, pp. 190-211, 2016.

[17] W. L. Chen and B. S. Jeng, "Analysis of piecewise constant delay systems via block-pulse functions," International Journal of Systems Science, vol. 12, no. 5, pp. 625-633, 1981.

[18] W.-L. Chen and C.-H. Meng, "A general procedure of solving the linear delay system via block pulse functions," Computers and Electrical Engineering, vol. 9, no. 3-4, pp. 153-166, 1982.

[19] H. R. Marzban and M. Shahsiah, "Solution of piecewise constant delay systems using hybrid of block-pulse and Chebyshev polynomials," Optimal Control Applications and Methods, vol. 32, no. 6, pp. 647-659, 2011.

[20] H. R. Marzban and S. M. Hoseini, "Analysis of linear piecewise constant delay systems using a hybrid numerical scheme," Advances in Numerical Analysis, vol. 2016, Article ID 8903184, 9 pages, 2016.

[21] H. R. Marzban and S. M. Hoseini, "Numerical treatment of nonlinear optimal control problems involving piecewise constant delay," IMA Journal of Mathematical Control and Information, 2015.

[22] Z. H. Jiang and W. Schaufelberger, Block pulse functions and their applications in control systems, vol. 179 of Lecture Notes in Control and Information Sciences, Springer, Berlin, Germany, 1992.
[23] H. R. Marzban, "Parameter identification of linear multi-delay systems via a hybrid of block-pulse functions and Taylor's polynomials," International Journal of Control, 2016.

[24] P. Lancaster, Theory of matrices, Academic Press, New York, NY, USA, 1969. 


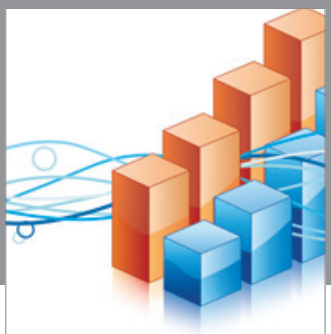

Advances in

Operations Research

vatem alat4

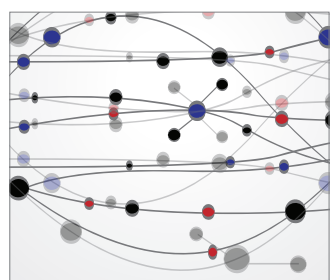

\section{The Scientific} World Journal
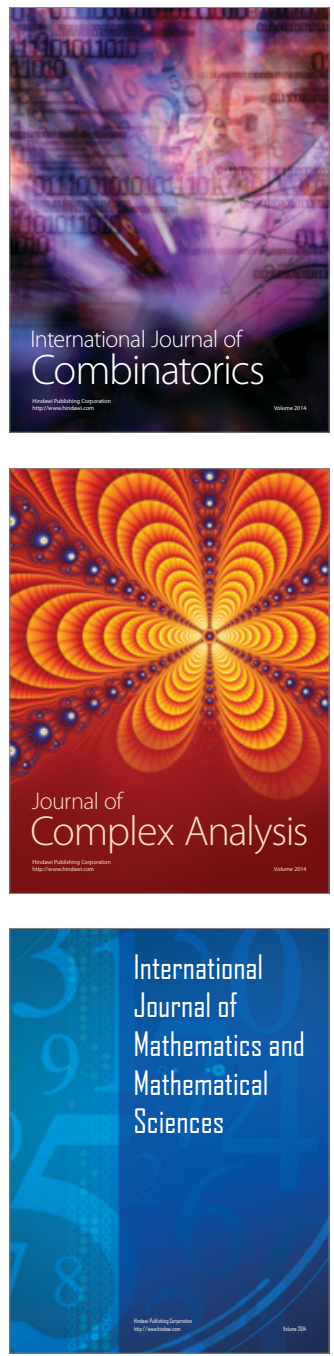
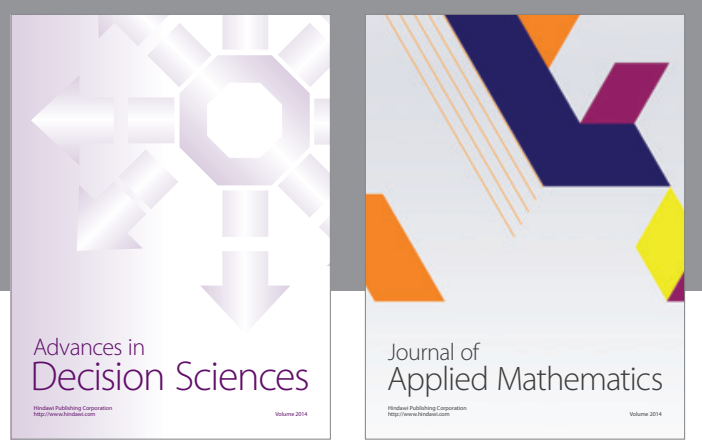

Algebra

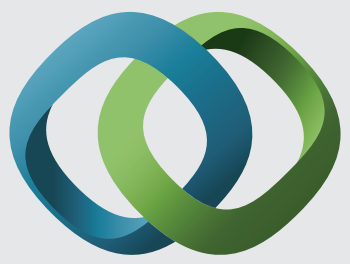

\section{Hindawi}

Submit your manuscripts at

http://www.hindawi.com
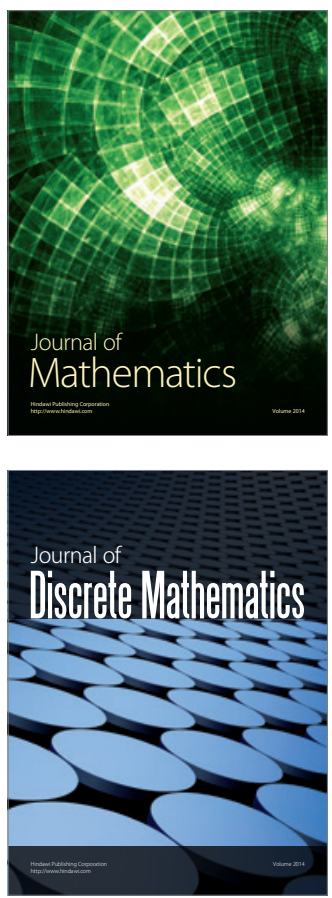

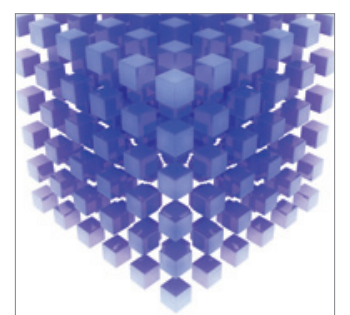

Mathematical Problems in Engineering
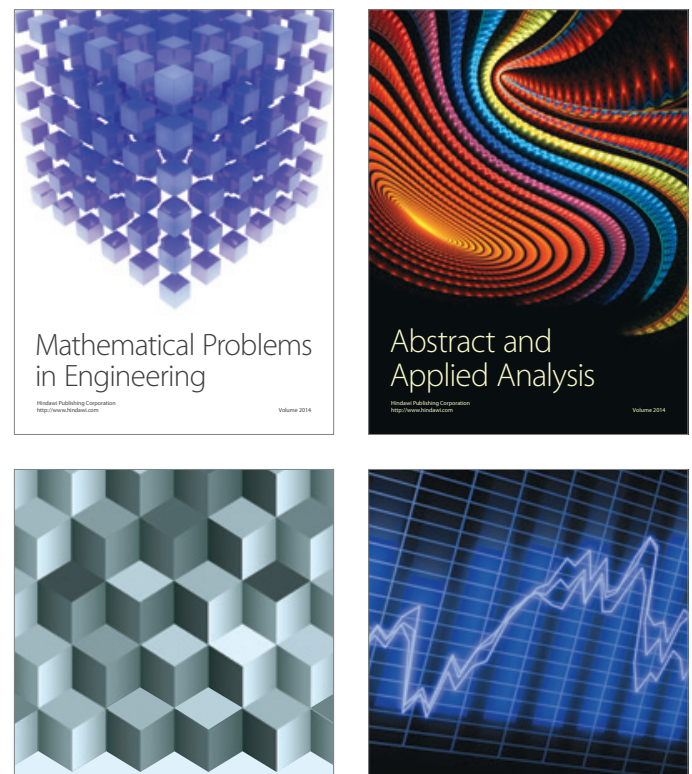

Journal of

Function Spaces

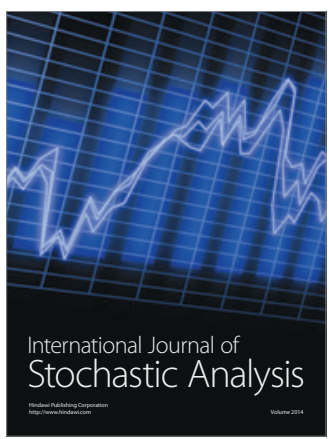

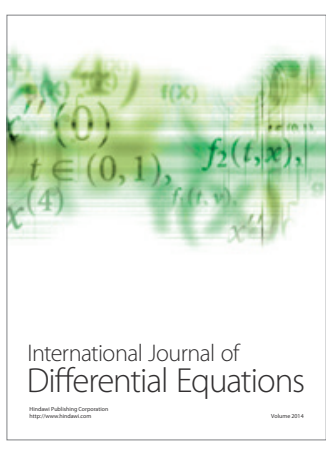
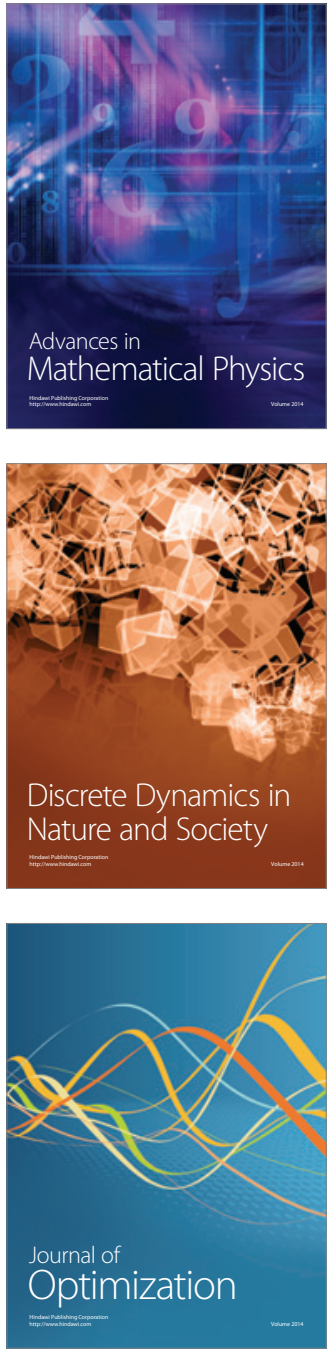